\title{
Food and nutrient intake of children and adolescents living in Germany: results from EsKiMo II
}

\author{
Clarissa Lage Barbosa, Anna-Kristin Brettschneider, Marjolein Haftenberger, \\ Franziska Lehmann, Almut Richter and Gert B.M. Mensink \\ Robert Kocht Institute, Berlin, Germany
}

\section{Abstract}

Introduction: An appropriate diet is particularly important for development, growth and health of children and adolescents. The living and social environments, as well as the food market and dietary habits have changed in recent decades. The second "Eating Study as a KiGGS Module" EsKiMo II (2015-2017) provides a current overview of the food and nutrient intake of children and adolescents living in Germany.

Material and Methods: About ten years after EsKiMo I (2006; N = 2,506), EsKiMo II (2015-2017) investigated the dietary behavior of 2,644 children and adolescents aged six to 17 years who previously participated in the second wave of the "German Health Interview and Examination Survey for Children and Adolescents" (KiGGS Wave 2) of the Robert Koch Institute. Food intake of the 6-11 year-olds was assessed by weighted food records on three consecutive days and one independent day. Adolescents (12-17 years) were interviewed about their food intake during the past four weeks using the Dietary Interview Software for Health Examination Studies (DISHES). Individual food group intake was compared with the food based dietary guidelines for children and adolescents in Germany. Energy and nutrient intake was calculated using the German Nutrient Database (BLS 3.02); intake from supplements was included. Individual intakes were compared with the Estimated Average Requirements (EAR) for Nordic countries.

Results: Most children and adolescents do not achieve the recommendation for fruits, vegetables, and milk/dairy products, whereas the maximal recommended intake of meat and "tolerated foods", such as sweets and soft-drinks, is exceeded by almost all children and adolescents.

The mean energy intake proportions $\left(\mathrm{En}^{\mathrm{O}} \%\right)$ for macronutrients correspond to the reference values. The majority of children and adolescents have intakes above the EAR for most micronutrients, except for iodine and vitamin D for all age groups and gender, and iron for the 12-17-year-old girls.

Discussion: EsKiMo II observed that the majority of the 6-17-year-olds is adequately supplied with macronutrients and most vitamins and minerals. Vitamin D and iodine supply are critical for all age groups and gender. However, there is much potential to improve food consumption patterns. These results are relevant for decision-making, and evaluation of nutrition, consumer and health policies.

Acknowledgements: The study is funded by the German Federal Ministry of Food and Agriculture through the Federal Office for Agriculture and Food.

\section{Conflict of Interest}

There is no conflict of interest. 